九州大学学術情報リポジトリ

Kyushu University Institutional Repository

\title{
UNLINKING SINGULAR LOCI FROM REGULAR FIBERS AND ITS APPLICATION TO SUBMERSIONS
}

Saeki, Osamu

Institute of Mathematics for Industry, Kyushu University

http://hdl. handle. net/2324/4485669

出版情報 : Journal of Singularities. 22, pp.92-103，2020. Worldwide Center of Mathematics, LLC バージョン：

権利関係 : 


\title{
UNLINKING SINGULAR LOCI FROM REGULAR FIBERS AND ITS APPLICATION TO SUBMERSIONS
}

\author{
OSAMU SAEKI \\ Dedicated to Professor Maria Aparecida Soares Ruas on the occasion of her 70th birthday

\begin{abstract}
Given a null-cobordant oriented framed link $L$ in a closed oriented 3-manifold $M$, we study the condition for the existence of a generic smooth map of $M$ to the plane that has $L$ as an oriented framed regular fiber such that the singular point set is unlinked with $L$. As an application, we give a singularity theoretical proof to the theorem, originally proved by Hector, Peralta-Salas and Miyoshi, about the realization of a link in an open oriented
\end{abstract} \\ 3-manifold as a regular fiber of a submersion to the plane.
}

\section{INTRODUCTION}

Let $M$ be a smooth closed oriented 3-dimensional manifold and $f: M \rightarrow \mathbf{R}^{2}$ a smooth map. If $y \in f(M) \subset \mathbf{R}^{2}$ is a regular value, then $f^{-1}(y)$ is an oriented link in $M$ and is naturally framed. Furthermore, if $f$ is generic enough, then the singular point set $S(f)$ of $f$ is an unoriented link in $M \backslash f^{-1}(y)$. In our previous paper [18], for an oriented framed link $L$ in $M$, we characterized those unoriented links in $M \backslash L$ which arise as the singular point set of a generic map that has $L$ as an oriented framed regular fiber. Such a characterization was given in terms of a relative Stiefel-Whitney class, or an obstruction to extending the trivialization of $\left.T M\right|_{L}$ induced by the framing over the whole manifold $M$.

In this paper, we first study the obstruction class more in detail, and give a more practical characterization in terms of $\mathbf{Z}_{2}$ linking numbers. We also clarify the components of $L$ which have non-trivial $\mathbf{Z}_{2}$ linking numbers with the singular point set. Then, as an application of such studies, we consider submersions of open oriented 3-manifolds to $\mathbf{R}^{2}$ that realize given oriented framed links as regular fibers. The idea is to consider a generic map $f$ whose singular point set $S(f)$ is unlinked with a given oriented framed regular fiber and to delete a neighborhood of the singular point set $S(f)$ for obtaining a submersion. In this way, we get a singularity theoretical proof to the characterization theorem, originally due to Hector and Peralta-Salas [8] and Miyoshi [13], of those oriented (framed) links in $\mathbf{R}^{3}$ that arise as regular fibers of submersions. Recall that their proofs used the h-principle for submersions due to Phillips [15]. Instead, in this paper, we arrange the singular point set by using Levine's cusp elimination techniques [11] (see also $[17,18]$ ) in a controlled way and push it to infinity, so that we get a submersion.

The paper is organized as follows. In $\S 2$, we recall several definitions and terminologies together with our main theorem in [18], which describes the characterization of singular point sets as unoriented links in terms of a certain obstruction class. In $\S 3$, we study the obstruction class more in detail, especially for closed oriented

Date: January 14, 2019.

2000 Mathematics Subject Classification. Primary 57R45; Secondary 57R30, 58K30, 57M25, $57 \mathrm{R} 20$.

Key words and phrases. Submersion, link, 3-manifold, excellent map, singular point set, regular fiber, relative Stiefel-Whitney class, framing. 
3-manifolds $M$ with $H_{*}(M ; \mathbf{Z}) \cong H_{*}\left(S^{3} ; \mathbf{Z}\right)$. In such a case, we can identify the obstruction class in terms of $\mathbf{Z}_{2}$ linking numbers. Then, we can describe the condition for the obstruction class to vanish in terms of $\mathbf{Z}_{2}$ linking numbers. Finally in $\S 4$, we apply these results to submersions of open oriented 3 -manifolds to $\mathbf{R}^{2}$. We will see that our singularity theoretical proof works well for punctured 3-manifolds, i.e. open 3-manifolds of the form $M^{\circ}=M \backslash D^{3}$ obtained from a closed 3-manifold $M$ by removing a small 3 -disk $D^{3}$ in $M$. For a general open oriented 3-manifold, we need to use an "absolute version" of the h-principle due to Phillips. Recall that the original proof due to Hector and Peralta-Salas [8] or Miyoshi [13] used the "relative version", stronger than the "absolute version", of the h-principle [6].

Throughout the paper, manifolds and maps are differentiable of class $C^{\infty}$ unless otherwise indicated. All (co)homology groups are with $\mathbf{Z}_{2}$-coefficients unless otherwise indicated. The symbol "”" means an appropriate isomorphism between algebraic objects or a diffeomorphism between smooth manifolds.

\section{Preliminaries}

Let $M$ (resp. $N$ ) be a closed 3-dimensional manifold (resp. a surface) and consider a map $f: M \rightarrow N$. We denote by $S(f)$ the set of singular points of $f$. A point in $S(f)$ is a fold singularity (or a cusp singularity) of $f$ if the map germ of $f$ at that point is modeled on the map germ $(x, y, z) \mapsto\left(x, y^{2} \pm z^{2}\right)$ (resp. $\left.(x, y, z) \mapsto\left(x, y^{3}+x y-z^{2}\right)\right)$ at the origin. We say that a fold singularity is definite (resp. indefinite) if it is modeled on the map germ $(x, y, z) \mapsto\left(x, y^{2}+z^{2}\right)$ (resp. $\left.(x, y, z) \mapsto\left(x, y^{2}-z^{2}\right)\right)$. We say that $f$ is excellent if $S(f)$ consists only of fold and cusp singularities. It is known that the set of excellent maps is always open and dense in the mapping space $C^{\infty}(M, N)$ endowed with the Whitney $C^{\infty}$ topology (for example, see [5, 20]). If $f$ is an excellent map, then $S(f)$ is an (unoriented) link in $M$, i.e. a finite disjoint union of smoothly embedded circles.

Let $f: M \rightarrow N$ be a map. For a regular value $y \in f(M) \subset N$, we call $L=f^{-1}(y)$ a regular fiber, which is a link in $M \backslash S(f)$. Note that $L$ is naturally framed: its framing is given as the pull-back of the trivial normal framing of the point $y$ in $N$. Furthermore, when $M$ and $N$ are oriented, $L$ is naturally oriented.

In the following, we fix an orientation for $\mathbf{R}^{2}$ once and for all. For excellent maps of closed oriented 3-manifolds into $\mathbf{R}^{2}$, we have the following (for details, see [16, Proposition 5.1] and [18]).

LEMMA 2.1. Let $L$ be an oriented framed link in a closed oriented 3-manifold $M$. Then, it is realized as an oriented framed regular fiber of an excellent map $f: M \rightarrow$ $\mathbf{R}^{2}$ if and only if it is framed null-cobordant: i.e. there exists a compact oriented normally framed surface $V$ embedded in $M$ whose framed boundary coincides with $L$.

REMARK 2.2. Let $L$ be an oriented link in a closed oriented 3-manifold $M$. Then, we can easily show that it bounds a compact oriented surface in $M$ if and only if $L$ represents zero in $H_{1}(M ; \mathbf{Z})$. This can be proved by considering a certain map $M \backslash L \rightarrow S^{1}$. In particular, if $H_{1}(M ; \mathbf{Z})=0$, then every oriented link bounds a compact oriented surface embedded in $M$.

REMARK 2.3. In [3], it is shown that every link in $S^{3}$ is a regular fiber of the restriction to $S^{3}$ of a certain polynomial map $\mathbf{C}^{2} \rightarrow \mathbf{C}$.

Now, let $L$ be an oriented framed link in a closed oriented 3 -manifold. If $L$ is realized as a framed regular fiber of an excellent map $f: M \rightarrow \mathbf{R}^{2}$, then $S(f)$ is a link in $M \backslash L$. Thus, it is natural to ask the following. 
Question 2.4. Which links in $M \backslash L$ appear as the singular point set $S(f)$ of an excellent map $f: M \rightarrow \mathbf{R}^{2}$ such that $f^{-1}(y)$ coincides with $L$ as oriented framed links for some regular value $y \in \mathbf{R}^{2}$ ?

In order to answer to the above question, let us prepare some notations and terminologies. For an (unoriented) link $J$ in $M \backslash L$, we denote by $[J]_{2} \in H_{1}(M \backslash L)$ the $\mathbf{Z}_{2}$-homology class represented by $J$. Let $N(L)$ be a small tubular neighborhood of $L$ in $M$ disjoint from $J$. Since $L$ is a framed link, we have a natural trivialization of $\left.T M\right|_{N(L)}$. The obstruction to extending it over $M$ is the relative Stiefel-Whitney class (see [9]), denoted by $w_{2}(M, L)$, which is an element of the $\mathbf{Z}_{2}$-cohomology group $H^{2}(M, N(L)) \cong H^{2}(M, L)$. Note that by excision and Poincaré-Lefschetz duality, we have

$$
H^{2}(M, N(L)) \cong H^{2}(M \backslash \operatorname{Int} N(L), \partial N(L)) \cong H_{1}(M \backslash \operatorname{Int} N(L)) \cong H_{1}(M \backslash L) .
$$

The following characterization, which answers to Question 2.4, has been proved in [18]. Recall that the proof was singularity theoretical in the sense that we used a result of Thom [19] about the homology class represented by the singular locus, and a cusp elimination result by Levine [11] for arranging the singular locus of an excellent map.

THEOREM 2.5. Let $L$ be an oriented null-cobordant framed link in a closed oriented 3-manifold $M$, and $J$ an unoriented link in $M \backslash L$. Then, there exist an excellent map $f: M \rightarrow \mathbf{R}^{2}$ and a regular value $y \in \mathbf{R}^{2}$ such that $f^{-1}(y)$ coincides with $L$ as oriented framed links and that $S(f)=J$ if and only if $[J]_{2} \in H_{1}(M \backslash L)$ is Poincaré dual to $w_{2}(M, L) \in H^{2}(M, L)$.

\section{Case of integral homology 3-SPheres}

In this section, we mainly consider closed oriented 3-manifolds $M$ with

$$
H_{*}(M ; \mathbf{Z}) \cong H_{*}\left(S^{3} ; \mathbf{Z}\right)
$$

and replace the condition described by the obstruction class $w_{2}(M, L)$ in Theorem 2.5 with that of $\mathbf{Z}_{2}$ linking numbers.

First, let $M$ be an arbitrary closed oriented 3-manifold and $L$ an oriented framed link in $M$. For the inclusion $j:(M, \emptyset) \rightarrow(M, L)$, the induced homomorphism $j^{*}: H^{2}(M, L) \rightarrow H^{2}(M)$ sends $w_{2}(M, L)$ to the second Stiefel-Whitney class $w_{2}(M)$ of $M$, which vanishes. By the cohomology exact sequence

$$
H^{1}(L) \stackrel{\delta}{\longrightarrow} H^{2}(M, L) \stackrel{j^{*}}{\longrightarrow} H^{2}(M),
$$

we have that $w_{2}(M, L)=\delta(\alpha)$ for some $\alpha \in H^{1}(L)$, although such an $\alpha$ may not be unique. In fact, such a class can be explicitly given as follows.

Set $L=L_{1} \cup L_{2} \cup \cdots \cup L_{\mu}$, where $L_{s}$ are the components of $L, s=1,2, \ldots, \mu$. It is known that the tangent bundle $T M$ of a closed oriented 3-manifold $M$ is always trivial. Once a trivialization $\tau$ of $T M$ is fixed, we can compare it with the specific trivialization of $\left.T M\right|_{L_{s}}$ associated with the framing given for each component $L_{s}$ of the framed link $L$. (We consider the trivialization given by the ordered vector fields $v_{1}, v_{2}$ and $v_{3}$, where $v_{1}$ is tangent to $L_{s}$ consistent with the orientation, and $v_{2}, v_{3}$ are consistent with the framing.) This defines a well-defined element $a_{s}$ in $\pi_{1}(S O(3)) \cong \mathbf{Z}_{2}$ for each $s$. Then, we have proved the following in [18].

LEMMA 3.1. Let $\alpha \in H^{1}(L)$ be the unique cohomology class such that the Kronecker product $\left\langle\alpha,\left[L_{s}\right]_{2}\right\rangle \in \mathbf{Z}_{2}$ coincides with $a_{s}$ for each component $L_{s}$ of $L$. Then, we have $\delta(\alpha)=w_{2}(M, L)$.

Note that the trivialization $\tau$ of $T M$ may not be unique. The set of homotopy classes of such trivializations is in one-to-one correspondence with the homotopy 
set $[M, S O(3)]$. If we consider the set of homotopy classes of trivializations on the 2-skeleton of $M$, then each such trivialization up to homotopy defines a spin structure on $M$, and the set of spin structures is in one-to-one correspondence with $H^{1}(M)$ (see [12]).

By the cohomology exact sequence,

$$
H^{1}(M) \stackrel{i^{*}}{\longrightarrow} H^{1}(L) \stackrel{\delta}{\longrightarrow} H^{2}(M, L) \stackrel{j^{*}}{\longrightarrow} H^{2}(M),
$$

we see that for an arbitrary element $\beta \in \operatorname{Im} i^{*}$, we could choose $\alpha+\beta$ instead of $\alpha$, where $i: L \rightarrow M$ is the inclusion map. The observation in the previous paragraph shows that this corresponds to choosing another trivialization which is "twisted along $\beta "$.

The following proposition has also been proved in [18].

LEMMA 3.2. Let $L$ be an oriented framed link which bounds a compact oriented surface $V$ consistent with the framing. Let $\alpha \in H^{1}(L)$ be an element such that $\delta(\alpha)=w_{2}(M, L)$. Then, we have

$$
\begin{aligned}
\left\langle w_{2}(M, L),[V, \partial V]_{2}\right\rangle & =\left\langle\delta(\alpha),[V, \partial V]_{2}\right\rangle \\
& =\left\langle\alpha,[L]_{2}\right\rangle \\
& \equiv \sharp L(\bmod 2),
\end{aligned}
$$

where $\langle\cdot, \cdot\rangle$ is the Kronecker product, $[V, \partial V]_{2} \in H_{2}(M, L)$ is the fundamental class of $V$ in $\mathbf{Z}_{2}$-coefficients, and $\sharp L$ denotes the number of components of $L$.

Note that the above lemma is applicable for an arbitrary null-cobordant framed link $L$ and that the value $\left\langle\alpha,[L]_{2}\right\rangle \in \mathbf{Z}_{2}$ does not depend on a particular choice of $\alpha$. Furthermore, if $L$ has an odd number of components, then the obstruction $w_{2}(M, L)$ never vanishes.

Let us now consider the case of a local knot component. Suppose that the oriented framed link $L$ contains a component $L_{s}$ that lies in the interior of a closed 3 -disk $D$ embedded in $M$. Set $U=\operatorname{Int} D$, which is an open set of $M$ diffeomorphic to $\mathbf{R}^{3}$. In the following, let us identify $U$ with $\mathbf{R}^{3}$. In this case, up to homotopy, we may assume that the trivialization $\tau$ of $T M$ over $U$ is given by the standard one of $T \mathbf{R}^{3}$.

Let $\pi: \mathbf{R}^{3} \rightarrow H$ be the orthogonal projection onto a generic hyperplane $H \cong \mathbf{R}^{2}$ in the sense that $\left.\pi\right|_{L_{s}}$ is an immersion with normal crossings. Recall that the first vector field defining the trivialization $\left.T M\right|_{L_{s}}$ associated with the framing on $L_{s}$ is tangent to $L_{s}$ consistent with the orientation. Since $\left.\pi\right|_{K}$ is an immersion, we may assume that at each point $x$ of $L_{s}$ the remaining two vector fields give a 2framing that is a basis for a 2-plane $N_{x} \subset T_{x} \mathbf{R}^{3}$ transverse to $T_{x} L_{s}$ containing the direction $H^{\perp}$ perpendicular to $H$. Then, we count the number of times modulo 2 the 2 -framing rotates in $N_{x}$ with respect to a fixed positive direction of $H^{\perp}$ while $x$ goes once around $L_{s}$. This number is denoted by $t_{v}\left(L_{s}\right)$, which is an element in $\mathbf{Z}_{2}$. Then, we have proved the following in [18].

Lemma 3.3. Let $\alpha \in H^{1}(L)$ be an arbitrary element such that $\delta(\alpha)=w_{2}(M, L)$. Then, we have

$$
\left\langle\alpha,\left[L_{s}\right]_{2}\right\rangle \equiv t_{v}\left(L_{s}\right)+c\left(L_{s}\right)+1 \quad(\bmod 2),
$$

where $c\left(L_{s}\right)$ denotes the number of crossings of the immersion $\left.\pi\right|_{L_{s}}: L_{s} \rightarrow H$ with normal crossings.

From now on, we will consider integral homology 3 -spheres for $M$ in this section. Let us start with the following.

Definition 3.4. For an oriented link $L$ in a closed oriented 3-manifold $M$ with $H_{1}(M ; \mathbf{Z})=0$, we always have a Seifert surface, i.e. a compact oriented surface $V$ 
positive
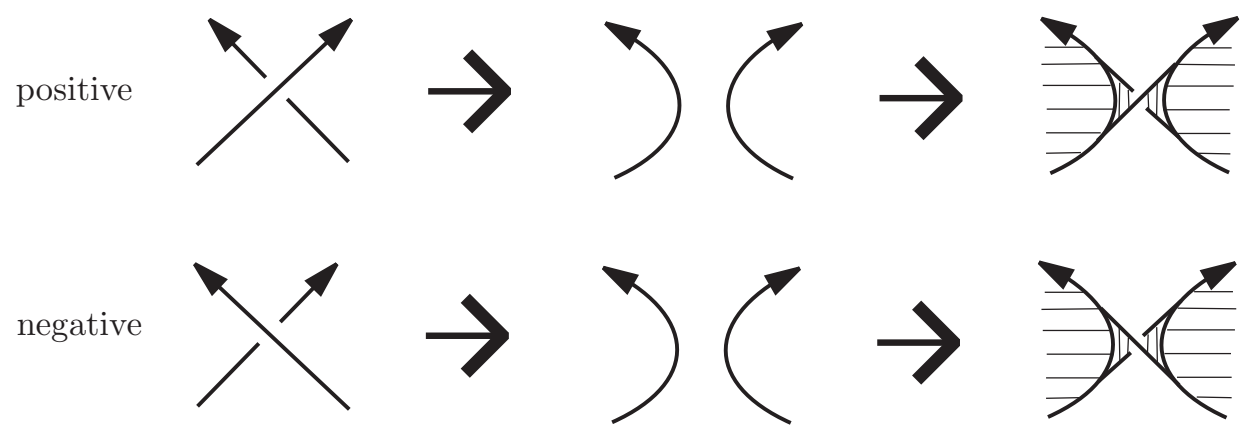

FiguRE 1. Seifert algorithm for positive and negative crossings

embedded in $M$ such that $\partial V=L$. Such a Seifert surface is not unique; however, it is known that the induced framing on $L$ is uniquely determined (for example, see $[8, \S 3.6 .1])$. In the following, such a framing is said to be preferred.

Then, for oriented links with preferred framings in the 3 -sphere $S^{3}$, we have the following. In the following, we fix an orientation for $S^{3}$ once and for all.

Proposition 3.5. Let $L=L_{1} \cup L_{2} \cup \cdots \cup L_{\mu}$ be an oriented link in $S^{3}$, on which a preferred framing is given. Then $w_{2}\left(S^{3}, L\right)=0$ if and only if for each $s$ with $1 \leq s \leq \mu$, we have

$$
\sum_{t \neq s} \operatorname{lk}\left(L_{s}, L_{t}\right) \equiv 1 \quad(\bmod 2)
$$

where $\mathrm{lk}$ denotes the linking number.

Proof. First, note that by the exact sequence (3.1) with $M=S^{3}$, we see that $\delta$ is injective and that $\alpha \in H^{1}(L)$ with $\delta(\alpha)=w_{2}\left(S^{3}, L\right)$ is uniquely determined. Therefore, $w_{2}\left(S^{3}, L\right)=0$ if and only if $\left\langle\alpha,\left[L_{s}\right]_{2}\right\rangle=0$ for all $s$.

Now, we may assume that $L$ is contained in $U \subset S^{3}$ as above, and let us consider the generic projection $\left.\pi\right|_{L}: L \rightarrow H$. By the so-called Seifert algorithm, we can construct a compact oriented surface $V \subset S^{3}$ with $\partial V=L$ (see Fig. 1). Then, by construction, we see that when $\pi(x)$ goes once around $\pi\left(L_{s}\right)$, each time it goes through a positive (resp. negative) crossing point, it contributes $+1 / 2($ resp. $-1 / 2)$ to $t_{v}\left(L_{s}\right)$. Since the number of crossing points of $\pi\left(L_{s}\right)$ and $\pi\left(L_{t}\right)$ is even for each $t \neq s$, and $\pi(x)$ goes through each self-crossing point of $\pi\left(L_{s}\right)$ twice, we have

$$
t_{v}\left(L_{s}\right) \equiv \frac{1}{2} \sum_{t \neq s} \widetilde{c}\left(L_{s}, L_{t}\right)+\widetilde{c}\left(L_{s}\right) \quad(\bmod 2)
$$

for each $s$, where $\widetilde{c}\left(L_{s}, L_{t}\right)$ is the sum of the signs of crossing points of $\pi\left(L_{s}\right)$ and $\pi\left(L_{t}\right)$, and $\widetilde{c}\left(L_{s}\right)$ is the sum of the signs of self-crossing points of $\pi\left(L_{s}\right)$. Then, since $\widetilde{c}\left(L_{s}\right) \equiv c\left(L_{s}\right)(\bmod 2)$, by Lemma 3.3 , we have

$$
\begin{aligned}
\left\langle\alpha,\left[L_{s}\right]_{2}\right\rangle & \equiv \frac{1}{2} \sum_{t \neq s} \widetilde{c}\left(L_{s}, L_{t}\right)+1 \quad(\bmod 2) \\
& \equiv \sum_{t \neq s} \operatorname{lk}\left(L_{s}, L_{t}\right)+1 \quad(\bmod 2)
\end{aligned}
$$

by the definition of linking numbers. Hence, the result follows.

In fact, we have the following more general result. 
Proposition 3.6. Let $M$ be a closed connected oriented 3-manifold with

$$
H_{1}(M ; \mathbf{Z})=0
$$

and $L=L_{1} \cup L_{2} \cup \cdots \cup L_{\mu}$ be an oriented link in $M$, on which a preferred framing is given. Then, $w_{2}(M, L)=0$ if and only if for each $s$ with $1 \leq s \leq \mu$, we have

$$
\sum_{t \neq s} \operatorname{lk}\left(L_{s}, L_{t}\right) \equiv 1 \quad(\bmod 2) .
$$

Proof. Since $H_{1}(M ; \mathbf{Z})=0$, there exists a Seifert surface $V$ for $L$, which is a compact oriented surface embedded in $M$ with $\partial V=L$. By definition, this is consistent with the framing of $L$. Set $V^{\prime}=V \backslash \operatorname{Int} N(L)$ and $\widetilde{L}_{s}=V^{\prime} \cap N\left(L_{s}\right)$ for each $s$, where $N(L)$ is a small tubular neighborhood of $L$ in $M, N\left(L_{s}\right)$ is the component of $N(L)$ containing $L_{s}, \partial N(L)$ intersects $V$ transversely, and $V \cap N(L)$ is a collar neighborhood of $\partial V$ in $V$. Note that $\widetilde{L}_{s}$ is a knot parallel to $L_{s}$, and we orient $\widetilde{L}_{s}$ consistently with $L_{s}$. Then, the oriented link $\widehat{L}_{s}=L \backslash L_{s}$ is $\mathbf{Z}$-homologous to $-\widetilde{L}_{s}$ in $M \backslash L_{s}$, where $-\widetilde{L}_{s}$ denotes $\widetilde{L}_{s}$ with the opposite orientation.

Now, suppose $w_{2}(M, L)=0$. In this case, the given framing of $L$ extends over $M$. Let us suppose that a Seifert surface $V_{s}$ for $L_{s}$ is consistent with the given framing of $L_{s}$ for some $s$. Then, by Lemma 3.2 applied to $L_{s}, w_{2}\left(M, L_{s}\right) \in H^{2}\left(M, L_{s}\right)$ does not vanish, as we obviously have $\sharp L_{s}=1$. This implies that $a_{s} \in \mathbf{Z}_{2}$ as appears in Lemma 3.1 does not vanish. This contradicts our assumption that the framing of $L$ extends over $M$. Therefore, an arbitrary Seifert surface $V_{s}$ for $L_{s}$ is not consistent with the given framing of $L_{s}$ for each $s$. Since $V$ is consistent with the framing of $L_{s}$, the linking number of $L_{s}$ and $\widetilde{L}_{s}$ must be an odd integer. Since $-\widetilde{L}_{s}$ is $\mathbf{Z}$-homologous to $\widehat{L}_{s}$ in $M \backslash L_{s}$, we have the congruence (3.2).

Conversely, suppose (3.2) holds for each $s$. Then, by the above argument we see that $a_{s}=0$ for each $s$. Hence, by Lemma 3.1, we have $w_{2}(M, L)=0$. This completes the proof.

In fact, the above argument implies the following.

Proposition 3.7. Let $M$ be a closed connected oriented 3-manifold with

$$
H_{1}(M ; \mathbf{Z})=0
$$

and $L=L_{1} \cup L_{2} \cup \cdots \cup L_{\mu}$ be an oriented link in $M$, on which a preferred framing is given. For each $s$ with $1 \leq s \leq \mu$, define $a_{s} \in \mathbf{Z}_{2}$ by

$$
a_{s}=\sum_{t \neq s} \operatorname{lk}\left(L_{s}, L_{t}\right)+1 \quad(\bmod 2) .
$$

Let $\alpha \in H^{1}(L)$ be the unique cohomology class such that $\left\langle\alpha,\left[L_{s}\right]_{2}\right\rangle=a_{s}$ for all $s$. Then, we have $\delta(\alpha)=w_{2}(M, L)$.

When $H_{1}(M ; \mathbf{Z})=0$, we have $H^{1}(M)=0=H^{2}(M)$, and hence the exact sequence (3.1) implies that we have the isomorphism $\delta: H^{1}(L) \rightarrow H^{2}(M, L)$. We easily see that its composition with the isomorphism $H^{2}(M, L) \rightarrow H_{1}(M-$ $L)$ corresponds to the Alexander duality whose inverse isomorphism is given by taking $\mathbf{Z}_{2}$ linking numbers. This observation together with Theorem 2.5 leads to the following, which answers to Question 2.4 for oriented framed links in integral homology 3-spheres.

THEOREM 3.8. Let $M$ be a closed connected oriented 3-manifold with

$$
H_{1}(M ; \mathbf{Z})=0,
$$

$L=L_{1} \cup L_{2} \cup \cdots \cup L_{\mu}$ be an oriented link in $M$, and $J$ be an unoriented link in $M \backslash L$. Then, there exists an excellent map $f: M \rightarrow \mathbf{R}^{2}$ such that $L=f^{-1}(y)$ for 
a regular value $y \in \mathbf{R}^{2}$ and $J=S(f)$ if and only if for each $s$ with $1 \leq s \leq \mu$, the $\mathbf{Z}_{2}$ linking number of $J$ with $L_{s}$ coincides with

$$
\sum_{t \neq s} \operatorname{lk}\left(L_{s}, L_{t}\right)+1 \quad(\bmod 2)
$$

Proof. By the above observations, we see that $[J]_{2} \in H_{1}(M \backslash L)$ is Poincaré dual to $w_{2}(M, L) \in H^{2}(M, L)$ if and only if it satisfies the condition on $\mathbf{Z}_{2}$ linking numbers in the theorem. Thus, the result follows from Theorem 2.5.

Let us observe the following.

LEMMA 3.9. If the congruence (3.2) holds, then the number of components of $L$ must be even.

Proof. Consider the sum of all linking numbers

$$
\sum_{s=1}^{\mu} \sum_{t \neq s} \operatorname{lk}\left(L_{s}, L_{t}\right) \in \mathbf{Z}
$$

over all $s$ and $t$ with $s \neq t$. Since $\operatorname{lk}\left(L_{s}, L_{t}\right)=\operatorname{lk}\left(L_{t}, L_{s}\right)$, the above sum must be even. On the other hand, the congruence (3.2) implies that the above sum has the same parity as the number of components of $L$. Thus the result follows.

The above lemma together with Theorem 3.8 implies that for an integral homology 3-sphere $M$ and an excellent map $f: M \rightarrow \mathbf{R}^{2}$, if $L=f^{-1}(y)$ has an odd number of components for a regular value $y \in \mathbf{R}^{2}$, then $S(f)$ has a non-trivial linking number with a component of $L$.

In order to get a more general result, let us introduce the following definition.

Definition 3.10. Let $M$ be a closed connected oriented 3-manifold and $L, L^{\prime}$ be non-empty disjoint links in $M$. We say that $L$ and $L^{\prime}$ are not linked if there exists an embedded 2-sphere in $M \backslash\left(L \cup L^{\prime}\right)$ which separates $M$ into two components in such a way that one of them contains $L$ and the other contains $L^{\prime}$. If such a 2 -sphere does not exist, then we say that $L$ and $L^{\prime}$ are linked.

LEMMA 3.11. Let $M$ be a closed connected oriented 3-manifold containing an embedded 2-sphere $S$ which separates $M$ into two components $M_{1}$ and $M_{2}$, where $M_{1}$ and $M_{2}$ are the closures of the connected components of $M \backslash S$. If a framed link $L$ is contained in Int $M_{1}$ and is framed null-cobordant in $M$, then it is also framed null-cobordant in Int $M_{1}$.

Proof. Let $V$ be a compact oriented normally framed surface in $M$ which bounds $L$ and is consistent with the framing of $L$. We may assume that $V$ and $S$ intersect each other transversely. Then, $V \cap S$ consists of a finite number of simple closed curves in the 2-sphere $S$. By considering $V \cap M_{1}$, adding 2 -disks bounded by the simple closed curves in $S$, and by slightly translating the 2 -disks in a parallel manner using the inner-most argument, we get a compact oriented surface embedded in $\operatorname{Int} M_{1}$. This gives a desired framed null-cobordism for $L$ in $\operatorname{Int} M_{1}$.

We have the following as a result of Lemma 3.11.

Proposition 3.12. Let $M$ be a closed connected oriented 3-manifold and $f: M \rightarrow$ $\mathbf{R}^{2}$ an excellent map. For a regular value $y \in \mathbf{R}^{2}$, if $L=f^{-1}(y)$ is non-empty and has an odd number of connected components, then $L$ is necessarily linked with $S(f)$.

Proof. Suppose that there exists a 2 -sphere $S$ that separates $L$ and $S(f)$. Let $M_{1}$ and $M_{2}$ be the closures of the two components of $M \backslash S$ such that $L \subset \operatorname{Int} M_{1}$ and $S(f) \subset$ Int $M_{2}$. Since $L$ is framed null-cobordant in $M$, it is also framed nullcobordant in Int $M_{1}$ by Lemma 3.11. Therefore, there exists a compact oriented 
normally framed surface in Int $M_{1}$ that bounds $L$. Let $\widehat{M}_{1}$ be the closed oriented 3-manifold obtained by attaching a 3 -disk to $M_{1}$ along the boundary $S$. Then, since $\left.f\right|_{M_{1}}$ is a submersion and $\pi_{2}(S O(3))$ vanishes, we see that the trivialization of $\left.T \widehat{M}_{1}\right|_{L}$ extends to $\widehat{M}_{1}$, and hence $w_{2}\left(\widehat{M}_{1}, L\right)$ vanishes. Then, by Lemma 3.2 applied to $L \subset \widehat{M_{1}}$, this leads to a contradiction, since $\sharp L$ is odd by our assumption. Therefore, $L$ and $S(f)$ are necessarily linked. This completes the proof.

In the case of integral homology 3 -spheres, by Theorem 3.8 we have the following.

Proposition 3.13. Let $M$ be a closed connected oriented 3-manifold with

$$
H_{1}(M ; \mathbf{Z})=0
$$

and $L=L_{1} \cup L_{2} \cup \cdots \cup L_{\mu}$ be an oriented link in $M$. For an arbitrary excellent map $f: M \rightarrow \mathbf{R}^{2}$ such that $L=f^{-1}(y)$ for a regular value $y \in \mathbf{R}^{2}, S(f)$ necessarily links with each component $L_{s}$ of $L$ with

$$
\sum_{t \neq s} \operatorname{lk}\left(L_{s}, L_{t}\right) \equiv 0 \quad(\bmod 2)
$$

Compare the above proposition with [18, Problem 5.1]. For example, if the congruence (3.3) holds for all $s$, then for an excellent map $f: M \rightarrow \mathbf{R}^{2}$ such that $f^{-1}(y)=L$ for a regular value $y \in \mathbf{R}^{2}$, each component of $L$ links with at least one component of $S(f)$.

We do not know if the results in this section for $M$ with $H_{1}(M ; \mathbf{Z})=0$ also hold for $M$ with $H_{1}(M)=0$ in $\mathbf{Z}_{2}$-coefficients.

\section{Submersions of open 3-MANifolds to $\mathbf{R}^{2}$}

In this section, as an application of our results in [18] and in the previous sections of the present paper, we consider submersions of open orientable 3 -manifolds to $\mathbf{R}^{2}$.

First, let us recall the following fundamental theorem for submersions of $\mathbf{R}^{3}$ to $\mathbf{R}^{2}$ obtained in [8].

TheOREM 4.1 (Hector and Peralta-Salas, 2012). Let $L=L_{1} \cup L_{2} \cup \cdots \cup L_{\mu} \subset \mathbf{R}^{3}$ be an oriented link in $\mathbf{R}^{3}$. Then, there exists a submersion $f: \mathbf{R}^{3} \rightarrow \mathbf{R}^{2}$ such that $f^{-1}(y)=L$ for some $y \in \mathbf{R}^{2}$ if and only if for each $s$ with $1 \leq s \leq \mu$, we have

$$
\sum_{t \neq s} \operatorname{lk}\left(L_{s}, L_{t}\right) \equiv 1 \quad(\bmod 2) \text {. }
$$

Recall that in [8], the authors used the h-principle for submersions $[6,15]$ for the proof. Here, we give a new proof to the above theorem using our singularity theoretical techniques.

Proof of Theorem 4.1. Let $L$ be an oriented link in $\mathbf{R}^{3}$ which satisfies the condition about the linking numbers as in the theorem. By identifying the interior of an embedded 3-disk $D$ in $S^{3}$ with $\mathbf{R}^{3}$, we may assume that $L \subset \operatorname{Int} D \subset S^{3}$. Then, by Proposition 3.5, we have $w_{2}\left(S^{3}, L\right)=0$ with respect to the preferred framing on $L$. Therefore, for an arbitrary non-empty link $J$ in $S^{3} \backslash D$, there exists an excellent map $g: S^{3} \rightarrow \mathbf{R}^{2}$ and a regular value $y \in \mathbf{R}^{2}$ such that $L=g^{-1}(y)$ and $J=S(g)$. By restricting $g$ to $\mathbf{R}^{3}=$ Int $D$, we get a submersion $f: \mathbf{R}^{3} \rightarrow \mathbf{R}^{2}$ which has $L$ as a regular fiber.

Conversely, suppose that we have a submersion $f: \mathbf{R}^{3} \rightarrow \mathbf{R}^{2}$ and a regular value $y \in \mathbf{R}^{2}$ such that $f^{-1}(y)=L$. Then, we can find an embedded 3-disk $D \subset \mathbf{R}^{3}$ whose interior contains $L$. Note that $\left.f\right|_{D}: D \rightarrow \mathbf{R}^{2}$ is a submersion which has $L$ as a regular fiber. By embedding $D$ into $S^{3}$, we can extend $\left.f\right|_{D}$ to a smooth map $g_{1}: S^{3} \rightarrow \mathbf{R}^{2}$. Here, $f(\partial D)$ misses $y \in \mathbf{R}^{2}$, and since the second homotopy group of $\mathbf{R}^{2} \backslash\{y\}$ is trivial, $\left.f\right|_{\partial D}$ is null-homotopic inside $\mathbf{R}^{2} \backslash\{y\}$. Therefore, we can 
arrange the smooth map $g_{1}$ in such a way that $g_{1}$ has $y \in \mathbf{R}^{2}$ as a regular value and that $g_{1}^{-1}(y)=L \subset$ Int $D$. Then, by slightly perturbing $g_{1}$ on a neighborhood of $S^{3} \backslash$ Int $D$, we get an excellent map $g_{2}: S^{3} \rightarrow \mathbf{R}^{2}$ such that $y \in \mathbf{R}^{2}$ is a regular value, that $g_{2}^{-1}(y)=L$, and that $S\left(g_{2}\right)$ is contained in $S^{3} \backslash$ Int $D$. In particular, $S\left(g_{2}\right)$ is $\mathbf{Z}_{2}$ null-homologous in $S^{3} \backslash L$, and hence we have $w_{2}\left(S^{3}, L\right)=0$. Then, by Proposition 3.5, we get the result.

REMARK 4.2. More generally, instead of $\mathbf{R}^{3}$, the above theorem holds also for an arbitrary open 3-manifold of the form $M \backslash D^{3}$ for a closed connected orientable 3 -dimensional manifold $M$ with $H_{1}(M ; \mathbf{Z})=0$, where $D^{3}$ is a small closed 3 -disk embedded in $M$.

In the case of a link with an odd number of components, we have the following.

REMARK 4.3. Let $f: \mathbf{R}^{3} \rightarrow \mathbf{R}^{2}$ be an excellent map, and suppose that $y \in \mathbf{R}^{2}$ is a regular value such that $L=f^{-1}(y)$ is compact and has an odd number of components. Then, by Proposition 3.12 together with an argument similar to the above, we see that the singular point set $S(f)$ necessarily links with $L$ (see also the paragraph just after [14, Theorem 10]): in other words, we can find no 2-sphere embedded in $\mathbf{R}^{3}$ that separates $L$ and $S(f)$. This implies, in particular, that such an $f$ can never be a submersion.

In fact, we have the following.

Proposition 4.4. Let $M$ be a closed connected orientable 3-manifold with

$$
H_{1}(M ; \mathbf{Z})=0
$$

and set $M^{\circ}=M \backslash D^{3}$. Let $L=L_{1} \cup L_{2} \cup \cdots \cup L_{\mu} \subset M^{\circ}$ be an oriented link such that $f^{-1}(y)=L$ for some excellent map $f: M^{\circ} \rightarrow \mathbf{R}^{2}$ and a regular value $y \in \mathbf{R}^{2}$. Then, each component $L_{s}$ of $L$ with

$$
\sum_{t \neq s} \operatorname{lk}\left(L_{s}, L_{t}\right) \equiv 0 \quad(\bmod 2)
$$

links with at least one component of $S(f)$. In particular, such an $f$ can never be a submersion.

Compare the above proposition with [18, Problem 5.1]. See also $[1,2,4,10]$ for related physical results.

Proof of Proposition 4.4. First note that each component of $S(f)$ is diffeomorphic to a circle or a real line. Furthermore, $S(f)$ is a closed submanifold of $M^{\circ}$ which may have infinitely many connected components.

Let $V_{s}$ be a Seifert surface for $L_{s}$ in $M$, where $L_{s}$ satisfies (4.1). We may assume that $L_{s} \subset M^{\circ}$ and that $S(f)$ intersects $L_{s}$ transversely at finitely many points. We have only to show that there are an odd number of intersection points.

Let $\widetilde{D}$ be a 3 -disk in $M$ such that Int $\widetilde{D} \supset D^{3}, L \cap \widetilde{D}=\emptyset, V_{s} \cap \widetilde{D}=\emptyset$, and that $\partial \widetilde{D}$ intersects $S(f)$ transversely at finitely many points. Then, by an argument similar to that in the proof of Theorem 4.1, we can construct an excellent map $g: M \rightarrow \mathbf{R}^{2}$ such that $\left.g\right|_{M \backslash \text { Int } \widetilde{D}}=\left.f\right|_{M \backslash \text { Int } \widetilde{D}}$ and that $g^{-1}(y)=L$. By our assumption (4.1), we have that $L_{s}$ has a non-trivial $\mathbf{Z}_{2}$ linking number with $S(g)$ by Theorem 3.8. Therefore, $S(g)$ intersects $V_{s}$ transversely at an odd number of points. By construction of $g$, this implies that $S(f)$ also intersects $V_{s}$ transversely at an odd number of points. This completes the proof.

The following is a special case of a theorem proved by Miyoshi [13], who used a relative version of the h-principle for submersions [6]. Here, we use our singularity theoretical arguments in order to prove the theorem for punctured 3-manifolds. 
THEOREM 4.5. Let $M$ be a closed orientable 3-manifold and $L$ a compact oriented framed link in $M^{\circ}=M \backslash D^{3}$. Then, there exists a submersion $f: M^{\circ} \rightarrow \mathbf{R}^{2}$ such that $f^{-1}(y)$ coincides with $L$ as oriented framed links for some $y \in \mathbf{R}^{2}$ if and only if $L$ bounds a proper normally framed surface in $M^{\circ}$ and the trivialization of $\left.T M^{\circ}\right|_{L}$ induced by the framing of $L$ extends over $M^{\circ}$.

Proof. If there exists a submersion $f$ as in the theorem, then the inverse image by $f$ of the half line $\left[y_{1}, \infty\right) \times\left\{y_{2}\right\} \subset \mathbf{R}^{2}$ is a proper normally framed surface in $M^{\circ}$ that bounds $L$, where $y=\left(y_{1}, y_{2}\right)$. Furthermore, since $f$ is a submersion, we can pull-back the natural trivialization of $T \mathbf{R}^{2}$ to $M^{\circ}$ by $f$ in such a way that the pull-back naturally extends the trivialization of $\left.T M^{\circ}\right|_{L}$ induced by the framing of $L$.

Conversely, suppose that $L$ bounds a proper normally framed surface $V$ in $M^{\circ}$ and the trivialization of $T M^{\circ}{ }{ }_{L}$ induced by the framing of $L$ extends over $M^{\circ}$. Let $\widetilde{D}$ be a small 3-disk neighborhood of $D^{3}$ whose interior contains $D^{3}$ such that $\widetilde{D} \subset M \backslash N(L)$ for a small tubular neighborhood $N(L)$ of $L$ in $M$. Then, we may assume that $V$ intersects $\partial \widetilde{D}$ transversely along finitely many embedded circles. Note that then $V \cap \partial \widetilde{D}$ bounds a compact oriented surface $V^{\prime}$ in $\widetilde{D}$. Then, by replacing $V \cap \widetilde{D}$ by $V^{\prime}$, we see that $L$ is framed null-cobordant in $M$. Furthermore, by our assumption, the trivialization of $\left.T M^{\circ}\right|_{L}$ induced by the framing of $L$ extends over $M^{\circ}$. Since $\pi_{2}(S O(3))$ vanishes, this implies that it also extends over $M$. Therefore, we have that the obstruction $w_{2}(M, L)$ vanishes. Hence, by Theorem 2.5, there exists an excellent map $f: M \rightarrow \mathbf{R}^{2}$ and a regular value $y \in \mathbf{R}^{2}$ such that $f^{-1}(y)$ coincides with $L$ as oriented framed links and that $S(f)$ is contained in Int $D^{3}$. Then, $f$ restricted to $M^{\circ}=M \backslash D^{3}$ is a desired submersion.

In fact, if we use the "absolute version" of the h-principle [15] in order to treat the end of an open 3-manifold, we can prove the following. Note again that the following theorem was originally proved by Miyoshi [13] by using a "relative version" of the h-principle [6].

THEOREM 4.6. Let $M$ be an open orientable 3-manifold and $L$ a compact oriented framed link in $M$. Then, there exists a submersion $f: M \rightarrow \mathbf{R}^{2}$ such that $f^{-1}(y)$ coincides with $L$ as oriented framed links for some $y \in \mathbf{R}^{2}$ if and only if $L$ bounds a proper normally framed surface in $M$ and the trivialization of $\left.T M\right|_{L}$ induced by the framing of $L$ extends over $M$.

Proof. Necessity can be proved by the same argument as in the proof of Theorem 4.5 .

Conversely, suppose that there exists a proper normally framed surface $V$ in $M$ that bounds $L$ as described in the theorem. Let $Q$ be a compact 3-dimensional submanifold of $M$ with boundary such that $\operatorname{Int} Q \supset L$ and that $\partial Q$ intersects $V$ transversely along finitely many embedded circles.

Let us first construct a smooth map $g_{1}: M \rightarrow \mathbf{R}^{2}$ as follows. Let $h: V \rightarrow[0, \infty)$ be a smooth function such that $h^{-1}(0)=\partial V=L$ and that $h$ is non-singular near $\partial V$. Let $N(V) \cong V \times I$ be a tubular neighborhood of $V$ in $M$, where $I=[-1,1]$ and the $I$-factor is consistent with the normal orientation of $V$. Then, we define $g_{1}$ on $N(V)$ by

$$
N(V) \cong V \times I \stackrel{h \times \operatorname{id}_{I}}{\longrightarrow}[0, \infty) \times I \subset \mathbf{R}^{2},
$$

where $\mathrm{id}_{I}$ is the identity map of $I$. We can extend $\left.g_{1}\right|_{N(V)}$ to $N(V) \cup N(L)$ in such a way that $\left.g_{1}\right|_{N(L)}$ is a submersion, that the origin 0 is a regular value, and that the framed regular fiber $g_{1}^{-1}(0)$ coincides with $L$. Then, since $\mathbf{R}^{2} \backslash g_{1}(N(V) \cup N(V))$ is contractible, we can extend $g_{1}$ to the whole manifold $M$ in such a way that 0 is still a regular value and that the framed regular fiber $g_{1}^{-1}(0)$ coincides with $L$. 
Set $Q^{\prime}=Q \backslash \operatorname{Int} N(L)$, which is a compact 3-manifold with boundary $\partial Q \cup$ $\partial N(L)$. Note that $g_{1}\left(Q^{\prime}\right) \subset \mathbf{R}^{2} \backslash$ Int $D$, where $D$ is a small 2-disk neighborhood of the origin.

By our assumption, the framing on $L$ extends over $M$. Using such a framing, we can construct a bundle epimorphism $T(M \backslash \operatorname{Int} Q) \rightarrow T\left(\mathbf{R}^{2} \backslash \operatorname{Int} D\right)$ covering $\left.g_{1}\right|_{M \backslash \text { Int } Q}$. Then, by the h-principle for submersions, $g_{1}$ is homotopic to a smooth map $g_{2}: M \rightarrow \mathbf{R}^{2}$ such that

(1) $g_{2}$ is a submersion over $M \backslash \operatorname{Int} Q$,

(2) $g_{2}=g_{1}$ over $N(L)$,

(3) $g_{2}(M \backslash \operatorname{Int} N(L)) \subset \mathbf{R}^{2} \backslash \operatorname{Int} D$.

Then, we can approximate $g_{2}$ by an excellent map $g_{3}$ that enjoys the same properties as $g_{2}$ described above. Then, $S\left(g_{3}\right)$ is a closed subset of $Q$, which is compact. Therefore, $S\left(g_{3}\right)$ is an unoriented link in $Q \backslash \operatorname{Int} N(L)$. Furthermore, as we started with a framing that extends over $M$, the obstruction to extending the framing on $\partial(Q \backslash \operatorname{Int} N(L))$ induced by $g_{3}$ to the whole $Q$ vanishes. This implies that the $\mathbf{Z}_{2}$-homology class represented by $S\left(g_{3}\right)$ vanishes in $Q$. Then, by our techniques developed in [18] using Levine's cusp eliminations (see $[11,17]$ ), we can homotope $g_{3}$ to an excellent map $g_{4}$ that satisfies the properties described above such that $S\left(g_{4}\right)$ is unlinked from $L$ : more precisely, there exists an embedded 3-disk $B \subset$ Int $Q \backslash N(L)$ such that Int $B \supset S\left(g_{4}\right)$. Then, for an appropriate embedded arc $A \subset M \backslash N(L)$ that "connects" $B$ to infinity, we see that $M$ is diffeomorphic to $M \backslash(A \cup B)$ by a diffeomorphism that is the identity on $N(L)$ (for example, see [13]). Then, the restriction of $g_{4}$ to $M \backslash(A \cup B)$ gives the desired submersion. This completes the proof.

REMARK 4.7. It is known that there exist open 3-manifolds that cannot be embedded in compact 3-manifolds [7].

We finish this paper by posing an open problem.

PROBLEM 4.8. Is there a polynomial map $\mathbf{R}^{3} \rightarrow \mathbf{R}^{2}$ that has a compact regular fiber as in Theorem 4.1 ?

Compare the above problem with Remark 2.3.

One can find some relevant open problems in $[8, \S 4]$ as well.

\section{ACKNOWLEDGMENT}

The author would like to express his sincere gratitude to Professor David Chillingworth for stimulating discussions and for indicating the works of Hector and Peralta-Salas $[8,14]$. The author has been supported in part by JSPS KAKENHI Grant Numbers JP16K13754, JP16H03936, JP17H06128.

\section{REFERENCES}

[1] M.V. Berry and M.R. Dennis, Knotted and linked phase singularities in monochromatic waves, Proc. R. Soc. Lond. A 457 (2001), 2251-2263.

[2] M.V Berry and M.R. Dennis, Topological events on wave dislocation lines: birth and death of loops, and reconnection, J. Physics A: Mathematical and Theoretical 40 (2007), 65-74.

[3] B. Bode and M.R. Dennis, Constructing a polynomial whose nodal set is any prescribed knot or link, preprint, arXiv:1612.06328 [math.GT].

[4] M.R. Dennis, Local phase structure of wave dislocation lines: twist and twirl, J. Opt. A: Pure Appl. Opt. 6 (2004), S202-S208.

[5] M. Golubitsky and V. Guillemin, Stable mappings and their singularities, Grad. Texts in Math., Vol. 14, Springer-Verlag, New York, Heidelberg, Berlin, 1973.

[6] A. Haefliger, Lectures on the theorem of Gromov, Proceedings of Liverpool Singularities Symposium, II (1969/1970) (Berlin), Lecture Notes in Math., vol. 209, Springer-Verlag, 1971, pp. $128-141$. 
[7] W. Haken, Some results on surfaces in 3-manifolds, in "1968 Studies in Modern Topology", pp. 39-98.

[8] G. Hector and D. Peralta-Salas, Integrable embeddings and foliations, Amer. J. Math. 134 (2012), 773-825.

[9] M.A. Kervaire, Relative characteristic classes, Amer. J. Math. 79 (1957), 517-558.

[10] H. Larocque, D. Sugic, D. Mortimer, A.J. Taylor, R. Fickler, R.W. Boyd, M.R. Dennis, and E. Karimi , Reconstructing the topology of optical polarization knots, Nature Physics (2018), https://doi.org/10.1038/s41567-018-0229-2.

[11] H.I. Levine, Elimination of cusps, Topology 3, Suppl. 2 (1965), 263-296.

[12] J. Milnor, Spin structures on manifolds, Enseignement Math. (2) 9 (1963), 198-203.

[13] S. Miyoshi, Links and submersions to the plane on an open 3-manifold, J. Topol. Anal. 8 (2016), 677-690.

[14] D. Peralta-Salas, Realization problems in the theory of foliations, In: S. Ibáñez, J.S. Pérez del Río, A. Pumariño, J.A. Rodríguez (eds.), Progress and Challenges in Dynamical Systems, Springer Proceedings in Mathematics \& Statistics, vol. 54, pp. 309-323, Springer, Berlin, Heidelberg, 2013.

[15] A. Phillips, Submersions of open manifolds, Topology 6 (1967), 171-206.

[16] O. Saeki, Stable maps and links in 3-manifolds, Kodai Math. J. 17 (1994), 518-529.

[17] O. Saeki, Constructing generic smooth maps of a manifold into a surface with prescribed singular loci, Ann. Inst. Fourier (Grenoble) 45 (1995), 1135-1162.

[18] O. Saeki, Linking between singular locus and regular fibers, preprint, arXiv:1804.00359 [math.GT].

[19] R. Thom, Les singularités des applications différentiables, Ann. Inst. Fourier, Grenoble 6 (1955-1956), 43-87.

[20] H. Whitney, On singularities of mappings of Euclidean spaces. I. Mappings of the plane into the plane, Ann. of Math. (2) 62 (1955), 374-410.

Institute of Mathematics for Industry, Kyushu University, Motooka 744, Nishi-Ku, FUKUOKA 819-0395, JAPAN

E-mail address: saeki@imi.kyushu-u.ac.jp 